\title{
NEGOCIAÇÃO DE CONFLITOS COMO COMPETÊNCIA DO ENFERMEIRO
}

\author{
NEGOTIATION OF CONFLICTS AS A \\ COMPETENCE OF THE NURSE
}

\section{LA NEGOCIACIÓN DE CONFLICTOS COMO UNA COMPETENCIA DEL ENFERMERO}

\author{
Denise Maria Osugui ${ }^{1}$ \\ Silvia Helena Henriques ${ }^{2}$ \\ Eliza Maria Rezende Dázio ${ }^{3}$ \\ Zélia Marilda Rodrigues Resck ${ }^{4}$ \\ Laura Andrian Leal ${ }^{5}$ \\ Roberta Seron Sanches ${ }^{6}$
}

Como citar este artigo: Osugui DM, Henriques SH, Dázio EMR, Resck ZMR, Leal LA, Sanches RS. Negociação de conflitos como competência do enfermeiro. Rev bahiana enferm. 2020;34:e-36035.

Objetivo: analisar a negociação de conflito enquanto competência profissional do enfermeiro no contexto hospitalar. Método: revisão integrativa da literatura. As bases de dados foram: PubMed, BDENF, CINAHL e LILACS no período de 2015 a 2019. Resultados: foram selecionados 17 artigos publicados. Dentre eles: 12 estudos qualitativos, 4 quantitativos e 1 estudo misto. Foram extraídas duas categorias temáticas: A negociação de conflito como competência do enfermeiro, que traz a gestão do conflito como fonte de sofrimento e a necessidade de interagir com outras competências para a efetiva gestão; e Estratégias negociadoras na resolução de conflitos, que apresenta formas de gerenciar um conflito. Conclusão: os estudos elegidos demonstraram que, no contexto hospitalar, a negociação de conflito, enquanto competência profissional do enfermeiro, é permeada pela insegurança.

Descritores: Conflito. Negociação. Enfermagem. Competência Profissional. Hospitais.

Objective: to analyze conflict negotiation as a professional competence of the nurse in the hospital context. Method: integrative literature review. The databases were: PubMed, BDENF, CINAHL and LILACS from 2015 to 2019. Results: 17 published articles were selected. Among them: 12 qualitative studies, 4 quantitative studies and 1 mixed study. Two thematic categories were extracted: The conflict negotiation as a competence of the nurse, which brings the management of the conflict as a source of suffering and the need to interact with other competencies for effective management; and Negotiating strategies in conflict resolution, which presents ways of managing a conflict. Conclusion: the studies elected demonstrated that, in the hospital context, conflict negotiation, as a professional competence of the nurse, is permeated by insecurity.

Descriptors: Conflict. Negotiation. Nursing. Professional Competence. Hospitals.

\footnotetext{
Enfermeira. Mestre em Enfermagem. Professora do Centro Universitário do Sul de Minas. Alfenas, Minas Gerais, Brasil. https://orcid.org/0000-0002-7452-4339.

Enfermeira. Doutora em Enfermagem. Professora Associada da Universidade de São Paulo. Ribeirão Preto, São Paulo, Brasil. https://orcid.org/0000-0003-2089-3304.

Enfermeira. Doutora em Enfermagem. Professora Associada da Universidade de São Paulo. Ribeirão Preto, São Paulo, Brasil. https://orcid.org/0000-000 I -92 I 6-6283.

Enfermeira. Doutora em Enfermagem. Professora Associada da Universidade Federal de Alfenas. Alfenas, Minas Gerais, Brasil. https://orcid.org/0000-0002-3752 8381.

5 Enfermeira. Mestre em Enfermagem. Universidade de São Paulo. Ribeirão Preto, São Paulo, Brasil. laura.andrian.leal@usp.br. https://orcid.org/0000-0002-85638980.

6 Enfermeira. Doutora em Enfermagem. Professora Adjunta da Universidade Federal de Alfenas. Alfenas, Minas Gerais, Brasil. https://orcid.org/0000-000 I-7557-5560.
} 
Objetivo: analizar la negociación de conflictos como competencia profesional de la enfermera en el contexto bospitalario. Método: revisión de la literatura integrativa. Las bases de datos fueron: PubMed, BDENF, CINAHL y LILACS en el periodo de 2015 a 2019. Resultados: Se seleccionaron 17 artículos publicados. Entre ellos: 12 estudios cualitativos, 4 cuantitativos y 1 mixto. Se extrajeron dos categorías temáticas: La negociación del conflicto como competencia del enfermero, que trae consigo la gestión de conflictos como fuente de sufrimiento y la necesidad de interactuar con otras competencias para una gestión eficaz; y Estrategias negociadoras en la resolución de conflictos, que presenta las formas de gestionar un conflicto. Conclusión: los estudios elegidos demostraron que, en el contexto hospitalario, la negociación de conflictos, como competencia profesional del enfermero, está permeada por la inseguridad.

Descriptores: Conflicto. Negociación. Enfermería. Competencia Profesional. Hospitales.

\section{Introdução}

A singularidade das organizações hospitalares tem sido destacada pela assistência a usuários em situações cada vez mais críticas. Este fato tem exigido dos profissionais de saúde que ali trabalham saberes específicos sobre sua área de atuação, a fim de enfrentarem as mudanças tecnológicas constantes, as condições de trabalho diversas (ora atuando no cuidado integral à beira do leito, ora atuando de maneira fragmentada), as alterações comportamentais manifestadas pela equipe de enfermagem, por meio do estresse - e os usuários cada vez mais exigentes. Esse enfrentamento diário tem provocado, muitas vezes, transformações no processo de trabalho, visto que a maneira de cuidar pode ser alterada conforme o modelo assistencial adotado. Em decorrência, a falta de entendimento da equipe de enfermagem pode gerar conflitos e ocasionar a desarmonia no ambiente de trabalho.

Conflito é uma palavra proveniente do latim, que remete à ideia de embate, discussão, desavença e oposição ${ }^{(1)}$. Na contemporaneidade, a abordagem gerencial reconhece que o conflito, nas organizações hospitalares, pode estar presente. O fato de se querer identificá-lo e abordá-lo é sinal de bem-estar organizacional da equipe de saúde, visto que a reflexão sobre este processo poderá trazer o entendimento entre as partes envolvidas, considerando que os conflitos podem estar relacionados à convivência entre pessoas de valores, crenças, formação e opiniões diferentes.

Sabe-se que o enfermeiro, no contexto hospitalar, possui diversas atribuições que se articulam com o cuidado direto com o usuário, bem como com a função gerencial ${ }^{(2)}$. Nessa direção, competências têm sido requeridas para este profissional, tais como: praticidade, informações, atitudes, valores, habilidades técnicas, raciocínio clínico e comunicação efetiva ${ }^{(3)}$.

Assim, o enfermeiro hospitalar tem que lidar com muitas demandas e tarefas com alto grau de exigências e responsabilidades que, dependendo de sua experiência e de seus saberes científicos e atitudes, podem favorecer ou não relações conflituosas nesse contexto de trabalho.

Nesse sentido, é essencial que o enfermeiro desenvolva aptidões também para a gestão de conflitos, a fim de garantir a qualidade no atendimento assistencial e a saúde organizacional. Para tanto, é imprescindível que tenha como destreza a habilidade de comunicação, observação, escuta, senso crítico e empatia para entrever todas as faces de um conflito ${ }^{(1)}$.

Estudo realizado com profissionais de enfermagem de um hospital público da região Nordeste do Brasil, com o objetivo de analisar a percepção da equipe de enfermagem sobre a função do gerente de enfermagem hospitalar, apontou que essa equipe é geradora de conflitos e gerenciá-los é um desafio para o enfermeiro ${ }^{(4)}$.

Nessa direção, este estudo apresenta os seguintes questionamentos: A negociação de conflitos é competência profissional do enfermeiro? Como se dá a negociação de conflitos realizada pelo enfermeiro no contexto do hospital?

Refletir sobre a negociação de conflitos pelo enfermeiro no contexto do hospital enquanto 
competência profissional justifica-se pelo fato de ser uma temática premente e que necessita de aprofundamento dos profissionais dessa área. Além disso, pelo fato de o contexto hospitalar revelar situações complexas, em que saber negociar conflitos trará resultados positivos tanto para o cuidado prestado ao usuário dos serviços quanto para a instituição.

Assim, o objetivo deste estudo é analisar a negociação de conflito enquanto competência profissional do enfermeiro no contexto hospitalar.

\section{Método}

Trata-se de uma revisão integrativa da literatura, método que permite analisar a bibliografia existente sobre determinado tema, obedecendo a padrões de rigor científico e metodológico ${ }^{(5)}$. Para tanto, foram percorridas seis etapas, como sugere estudo de revisão ${ }^{(6)}$ : identificação do tema e questão de pesquisa; amostragem ou busca dos estudos primários na literatura; determinação das informações a serem coletadas e categorização dos estudos selecionados; avaliação dos estudos inseridos na revisão; interpretação dos resultados; e apresentação da revisão ou síntese dos resultados.

A questão norteadora deste trabalho foi: "Quais são as evidências científicas disponíveis na literatura que tratam da temática da negociação de conflitos como competência do profissional enfermeiro no contexto hospitalar?" Para a construção da questão, foi utilizada a estratégia PICO, sendo P - população, paciente ou problema, o enfermeiro no contexto hospitalar; I - intervenção ou área de interesse, no caso as competências gerenciais do profissional; o elemento C - comparação entre intervenção ou grupo, o qual não foi empregado, devido ao tipo de revisão; para o elemento $\mathrm{O}$ - desfecho, foi considerada a negociação de conflitos ${ }^{(7)}$.

Para busca dos estudos primários, foram selecionadas e consultadas as seguintes bases de dados: National Library of Medicine National Institutes of Health (PubMed), Bases de dados da Enfermagem (Bdenf), Cumulative Index to Nursing and Allied Health Literature (CINAHL) e Literatura Latino-Americana e do Caribe em Ciências da Saúde (LILACS). Para tanto, foram utilizados os seguintes Descritores em Ciências da Saúde (DeCS) direcionados à temática proposta: PUBMED: Nursing/Conflict/Negotiating, Professional competence, hospitals; BDENF: Enfermagem, Nursing/Conflict, Conflito/Negociação, Negotiating, Competência profissional, Professional competence, Hospitais, Hospitals; CINAHL: Nursing/Conflict/Negotiating, Professional competence, hospitals; LILACS: Enfermagem, Conflito/Negociação, Competência profissional e Hospitais.

Considerando o cenário atual do setor saúde, marcado por elevadas demandas, competitividade, custo e exigências crescentes que podem contribuir para o desenvolvimento de conflito, os critérios de inclusão foram assim estabelecidos para a condução da revisão integrativa: estudos primários que abordavam as competências do profissional enfermeiro no gerenciamento de conflitos, publicados em inglês, espanhol e português, no período de janeiro de 2015 a setembro de 2019. Foram excluídos os estudos de revisão da literatura, editorial, teses, dissertações, livros e carta resposta. A busca dos estudos primários nas bases de dados ocorreu de setembro a outubro de 2019.

Com a finalidade de integrar os dados disponíveis, foi empregada a classificação de evidências, que determina o nível de evidência de acordo com a questão clínica utilizada no estudo: nível I - revisões sistemáticas com randomização; nível II - estudo clínico com randomização; nível III - estudo clinico sem randomização; nível IV - coorte e caso controle; nível V - revisão sistemática de estudos qualitativos; nível VI - estudos descritivos; e nível VII - opinião de especialistas ${ }^{(8)}$.

\section{Resultados e Discussão}

Identificou-se 2.455 estudos, dos quais 2.305 foram excluídos automaticamente por duplicidade e por estarem fora do período temporal proposto. Assim, obteve-se uma amostra de 150 estudos ao final da primeira etapa de avaliação 
dos artigos. Dentre estes, 37 (24,66\%) foram encontrados na LILACS; 14 (9,33\%), na CINAHL; 57 (38\%), na BDENF; 42 (28\%), na PUBMED. Na segunda etapa, procedeu-se à leitura completa dos 150 estudos, para identificar aqueles que respondiam satisfatoriamente à questão de pesquisa e/ou tinham pertinência com o objetivo do estudo. Desse processo, obteve-se uma amostra de 17 artigos incluídos, sendo 8 (47,05\%) da LILACS, $1(5,88 \%)$ da CINAHL, $6(35,29 \%)$ da BDENF e 2 $(11,76 \%)$ da PUBMED. O detalhamento das fases de pesquisa está apresentado no Fluxograma 1.

Fluxograma 1 - Fases da Revisão Integrativa

\begin{tabular}{|c|c|c|c|}
\hline & \multicolumn{2}{|c|}{ Estratégia de busca } & \\
\hline \multicolumn{4}{|l|}{ Coleta de dados } \\
\hline \multicolumn{4}{|c|}{ Total de 2.455 artigos } \\
\hline LILACS & CINAHL & BDENF & PUBMED \\
\hline \multirow[t]{2}{*}{873} & 291 & 443 & 848 \\
\hline & & \multicolumn{2}{|c|}{ Aplicação critérios de inclusão e exclusão } \\
\hline LILACS & CINAHL & BDENF & PUBMED \\
\hline \multirow[t]{2}{*}{37} & 14 & 57 & 42 \\
\hline & & \multicolumn{2}{|l|}{ Leitura na íntegra } \\
\hline LILACS & CINAHL & BDENF & PUBMED \\
\hline 8 & 1 & 6 & 2 \\
\hline $\begin{array}{l}7 \text { Português } \\
1 \text { Espanhol }\end{array}$ & 1 Português & $\begin{array}{l}3 \text { Português } \\
2 \text { Espanhol } \\
1 \text { Inglês }\end{array}$ & 2 Inglês \\
\hline
\end{tabular}

Fonte: Elaboração própria.

Foram selecionados 17 artigos publicados entre 2015 e 2019, sendo 4 em 2018, 2017 e 2016, 3 em 2019 e 2 em 2015. No que se refere à abordagem metodológica, 12 (70,58\%) publicações eram estudos qualitativos, dentre eles 2 em espanhol, 1 em inglês e 9 em português; os estudos quantitativos foram 4 (23,52\%), sendo 1 em espanhol, 2 em inglês e 1 em português; $1(0,05 \%)$ pesquisa mista em português. No que se refere ao nível de evidência, dentre os
17 estudos, 5 (29,5\%) foram classificados com tipo de questão clínica de Prognóstico/Predição ou Etiologia, todos com nível de evidência IV; $12(70,5 \%)$ foram classificados como questão clínica de Significado, nível de evidência II - Estudo clínico com randomização. Este resultado demonstra que a maioria apresenta forte nível de evidência. Os Quadros 1a e 1b apresentam a síntese da caracterização dos estudos.

Quadro 1a - Caracterização dos artigos selecionados segundo autores, título e periódico, ano, base de dados

\begin{tabular}{|l|l|l|}
\hline Autores & \multicolumn{1}{|c|}{ Título } & \multicolumn{1}{|c|}{$\begin{array}{c}\text { Periódico/Ano/ } \\
\text { Base de dados }\end{array}$} \\
\hline $\begin{array}{l}\text { Ramos FRS, Vargas MAO, } \\
\text { Schneider DG, Barlem ELD, } \\
\text { Scapin SQ, Schneider AMM }\end{array}$ & $\begin{array}{l}\text { Ethical conflict as a trigger for moral } \\
\text { suffering: survey of Brazilian nurses }\end{array}$ & $\begin{array}{l}\text { Rev enferm UERJ / } \\
2017 / \text { LILACS }\end{array}$ \\
\end{tabular}


Quadro 1a - Caracterização dos artigos selecionados segundo autores, título e periódico, ano, base de dados

(conclusão)

\begin{tabular}{|c|c|c|}
\hline Autores & Título & $\begin{array}{l}\text { Periódico/Ano/ } \\
\text { Base de dados }\end{array}$ \\
\hline Wood-Molina T, Rivas-Ribeiro $\mathrm{E}^{(10)}$ & $\begin{array}{l}\text { Conflictividad ética en enfermeras/os } \\
\text { de unidades de cuidados críticos en un } \\
\text { hospital del sur de Chile }\end{array}$ & $\begin{array}{l}\text { Enfermería } \\
\text { Universitaria / } 2017 \text { / } \\
\text { LILACS }\end{array}$ \\
\hline $\begin{array}{l}\text { Eduardo EA, Peres AM, } \\
\text { Kalinowski CE, Cunha ICKO, } \\
\text { Bernardino E }^{(11)}\end{array}$ & $\begin{array}{l}\text { The negotiator that we have and the } \\
\text { negotiator that we want in nursing }\end{array}$ & $\begin{array}{l}\text { Texto Contexto } \\
\text { Enferm / 2016 / } \\
\text { LILACS }\end{array}$ \\
\hline $\begin{array}{l}\text { Pinhatti EDG, Vannuchi MTO, } \\
\text { Sardinha DSS, Haddad MCL }\end{array}$ & $\begin{array}{l}\text { Job rotation of nursing professionals } \\
\text { among the sectors of a hospital: a } \\
\text { management tool in conflict resolution }\end{array}$ & $\begin{array}{l}\text { Texto Contexto } \\
\text { Enferm / 2017 / } \\
\text { LILACS }\end{array}$ \\
\hline $\begin{array}{l}\text { Leal LA, Soares MI, Silva BR, } \\
\text { Brito LJS, Bernardes A, } \\
\text { Henriques } \mathrm{SH}^{(13)}\end{array}$ & $\begin{array}{l}\text { Professional competencies for hospital } \\
\text { nurses: a documentary analysis }\end{array}$ & $\begin{array}{l}\text { Rev Enfermagem } \\
\text { Centro-Oeste Mineiro } \\
\text { / } 2019 \text { / LILACS } \\
\end{array}$ \\
\hline $\begin{array}{l}\text { Camelo SHH, Soares MI, } \\
\text { Chaves LDP, Rocha FLR, } \\
\text { Silva VLS }\end{array}$ & $\begin{array}{l}\text { Nurs } \\
\text { train }\end{array}$ & $\begin{array}{l}\text { Rev enferm UERJ / } \\
2016 \text { / LILACS }\end{array}$ \\
\hline $\begin{array}{l}\text { Leal LA, Soares MI, Silva BR, } \\
\text { Bernardes A, Camelo SHH }\end{array}$ & $\begin{array}{l}\text { Clinical and management skills for } \\
\text { hospital nurses: perspective of nursing } \\
\text { university students }\end{array}$ & $\begin{array}{l}\text { Rev Bras Enferm / } \\
2018 \text { / LILACS }\end{array}$ \\
\hline $\begin{array}{l}\text { Tironi NM, Bernardino E, } \\
\text { Haddad MCL, Nimtz MA, } \\
\text { Torres DG, Peres AM } \\
\end{array}$ & $\begin{array}{l}\text { Assignments and competencies of nursing } \\
\text { managers: a descriptive exploratory } \\
\text { research }\end{array}$ & $\begin{array}{l}\text { Online braz j nurs / } \\
2017 \text { / LILACS }\end{array}$ \\
\hline Grubaugh ML, Flyn L ${ }^{(17)}$ & $\begin{array}{l}\text { Relationships Among Nurse Manager } \\
\text { Leadership Skills, Conflict Management, } \\
\text { and Unit Teamwork. }\end{array}$ & $\begin{array}{l}\text { J Nursing Adm } \\
/ / 2018 \text { / CINAHL }\end{array}$ \\
\hline $\begin{array}{l}\text { Beserra EP, Gubert FM, } \\
\text { Martins MC, Vasconcelos VM, } \\
\text { Figueiredo GA, Silva LA, et al. }\end{array}$ & Conflict management in nurse training & $\begin{array}{l}\text { J Nurs UFPE online / } \\
2018 \text { / BDENF }\end{array}$ \\
\hline $\begin{array}{l}\text { Souza MS, Barlem JGT, } \\
\text { Hirsch CD, Rocha LP, } \\
\text { Neutzling BRS, Ramos AM }{ }^{(19)}\end{array}$ & $\begin{array}{l}\text { Dimensionamento e escalas de pessoal } \\
\text { de enfermagem: competências dos } \\
\text { enfermeiros }\end{array}$ & $\begin{array}{l}\text { Enferm Foco / } 2018 / \\
\text { BDENF }\end{array}$ \\
\hline $\begin{array}{l}\text { Amestoy SC, Peixoto RS, } \\
\text { Garcia RP, Santos BP, Silva CN, } \\
\text { Braga DD }^{(20)}\end{array}$ & $\begin{array}{l}\text { Percepção de enfermeiros-líderes sobre o } \\
\text { gerenciamento de conflitos no ambiente } \\
\text { hospitalar }\end{array}$ & $\begin{array}{l}\text { Rev enferm UFSM / } \\
2016 \text { / BDENF }\end{array}$ \\
\hline $\begin{array}{l}\text { Cordero-Maldonado E, } \\
\text { García-Domínguez JA, } \\
\text { Romero-Quechol GM, } \\
\text { Flores-Padilla L, Trejo-Franco J }\end{array}$ & $\begin{array}{l}\text { Dimensiones de la relación interpersonal } \\
\text { del profesional de enfermería en una } \\
\text { unidad de segundo nivel }\end{array}$ & $\begin{array}{l}\text { Rev Enferm Inst Mex } \\
\text { Seguro Soc/2019/ } \\
\text { BDENF }\end{array}$ \\
\hline $\begin{array}{l}\text { Santos JLG, Lima MADS, } \\
\text { Pestana AL, Colomé ICS, } \\
\text { Erdmann } \mathrm{AL}^{(22)}\end{array}$ & $\begin{array}{l}\text { Estratégias utilizadas pelos enfermeiros } \\
\text { para promover o trabalho em equipe em } \\
\text { um serviço de emergência }\end{array}$ & $\begin{array}{l}\text { Rev Gaúcha Enferm / } \\
2016 \text { / BDENF }\end{array}$ \\
\hline $\begin{array}{l}\text { Thofehrn MB, Montesinos MJL, } \\
\text { Jacondino MB, Fernandes } \mathrm{HN} \text {, } \\
\text { Gallo CMC, Figueira } \mathrm{AB}^{(23)}\end{array}$ & $\begin{array}{l}\text { Work process of nurse in health } \\
\text { production in a university hospital in } \\
\text { Múrcia/Espanha }\end{array}$ & $\begin{array}{l}\text { Cienc Cuid Saude / } \\
2015 \text { / BDENF }\end{array}$ \\
\hline Ika C, Novieastari E, Nuraini $\mathrm{T}^{(24)}$ & $\begin{array}{l}\text { The role of a head nurses in preventing } \\
\text { interdisciplinary conflicts }\end{array}$ & $\begin{array}{l}\text { Enferm Clin / 2019 / } \\
\text { PUBMED }\end{array}$ \\
\hline $\begin{array}{l}\text { Kim WS, Nicotera AM, } \\
\text { McNulty J }\end{array}$ & $\begin{array}{l}\text { Nurses' perceptions of conflict as } \\
\text { constructive or destructive }\end{array}$ & $\begin{array}{l}\text { J Adv Nurs / 2015/ } \\
\text { PUBMED }\end{array}$ \\
\hline
\end{tabular}

Fonte: Elaboração própria. 
Quadro 1b - Caracterização dos artigos selecionados segundo autores, delineamento do estudo, local e nível de evidência

\begin{tabular}{|c|c|c|c|}
\hline Autores & $\begin{array}{c}\text { Delineamento do } \\
\text { estudo }\end{array}$ & Local & $\begin{array}{c}\text { Nivel de } \\
\text { Evidência }\end{array}$ \\
\hline $\begin{array}{l}\text { Ramos FRS, Vargas MAO, } \\
\text { Schneider DG, Barlem ELD, } \\
\text { Scapin SQ, Schneider AMM }\end{array}$ & Estudo tipo Survey & $\begin{array}{l}\text { Capitais das } 27 \\
\text { Unidades Federativas } \\
\text { do Brasil }\end{array}$ & Nível II \\
\hline Wood-Molina T, Rivas-Ribeiro E $\mathrm{E}^{(10)}$ & Estudo quantitativo & Chile & Nível IV \\
\hline $\begin{array}{l}\text { Eduardo EA, Peres AM, } \\
\text { Kalinowski CE, Cunha ICKO, } \\
\text { Bernardino E }{ }^{(11)}\end{array}$ & Estudo qualitativo & Curitiba, Brasil & Nível II \\
\hline $\begin{array}{l}\text { Pinhatti EDG, Vannuchi MTO, } \\
\text { Sardinha DSS, Haddad MCL }\end{array}$ & Estudo qualitativo & Brasil & Nível II \\
\hline $\begin{array}{l}\text { Leal LA, Soares MI, Silva BR, } \\
\text { Brito LJS, Bernardes A, } \\
\text { Henriques } \mathrm{SH}^{(13)}\end{array}$ & Estudo qualitativo & Interior Paulista, Brasil & Nível II \\
\hline $\begin{array}{l}\text { Camelo SHH, Soares MI, } \\
\text { Chaves LDP, Rocha FLR, } \\
\text { Silva VLS }\end{array}$ & $\begin{array}{l}\text { Estudo quanti- } \\
\text { qualitativo }\end{array}$ & Interior Paulista, Brasil & Nível II \\
\hline $\begin{array}{l}\text { Leal LA, Soares MI, Silva BR, } \\
\text { Bernardes A, Camelo SHH }{ }^{(15)}\end{array}$ & Estudo qualitativo & Interior Paulista, Brasil & Nível II \\
\hline $\begin{array}{l}\text { Tironi NM, Bernardino E, } \\
\text { Haddad MCL, Nimtz MA, } \\
\text { Torres DG, Peres AM }\end{array}$ & Estudo qualitativo & Estado do Paraná, Brasil & Nível II \\
\hline Grubaugh ML, Flyn L ${ }^{(17)}$ & Estudo quantitativo & Colorado (EUA) & Nível IV \\
\hline $\begin{array}{l}\text { Beserra EP, Gubert FM, } \\
\text { Martins MC, Vasconcelos VM, } \\
\text { Figueiredo GA, Silva LA, et al. }^{(18)}\end{array}$ & Estudo reflexivo & Pernambuco, Brasil & Nível IV \\
\hline $\begin{array}{l}\text { Souza MS, Barlem JGT, Hirsch CD, } \\
\text { Rocha LP, Neutzling BRS, } \\
\text { Ramos AM }{ }^{(19)}\end{array}$ & Estudo quantitativo & Região Sul do Brasil & Nível IV \\
\hline $\begin{array}{l}\text { Amestoy SC, Peixoto RS, } \\
\text { Garcia RP, Santos BP, Silva CN, } \\
\text { Braga DD }\end{array}$ & Estudo qualitativo & Região Sul do Brasil & Nível II \\
\hline $\begin{array}{l}\text { Cordero-Maldonado E, } \\
\text { García-Domínguez JA, } \\
\text { Romero-Quechol GM, } \\
\text { Flores-Padilla L, Trejo-Franco J }\end{array}$ & Estudo descritivo & México & Nível IV \\
\hline $\begin{array}{l}\text { Santos JLG, Lima MADS, } \\
\text { Pestana AL, Colomé ICS, } \\
\text { Erdmann AL }\end{array}$ & Estudo qualitativo & Região do Sul do Brasil & Nível II \\
\hline $\begin{array}{l}\text { Thofehrn MB, Montesinos MJL, } \\
\text { Jacondino MB, Fernandes HN, } \\
\text { Gallo CMC, Figueira } \mathrm{AB}^{(23)}\end{array}$ & Estudo qualitativo & Murcia (Espanha) & Nível II \\
\hline Ika C, Novieastari E, Nuraini $T^{(24)}$ & Estudo qualitativo & Jacarta (Indonésia) & Nível II \\
\hline $\begin{array}{l}\text { Kim WS, Nicotera AM, } \\
\text { McNulty J }\end{array}$ & Estudo qualitativo & Espanha & Nível II \\
\hline
\end{tabular}

Fonte: Elaboração própria. 
A análise dos estudos possibilitou a construção de duas categorias, a saber: "Negociação de conflito como competência do enfermeiro" e "Estratégias negociadoras na resolução de conflitos".

\section{Negociação de conflito como competência do enfermeiro}

Observou-se, nos artigos que compõem a amostra, que o gerenciamento de conflitos é uma competência inerente ao trabalho do enfermeiro e contribui para o bem-estar da organização de saúde. Na organização hospitalar, o enfermeiro é considerado um gerenciador dos serviços de saúde. Ele desenvolve papel fundamental nas relações de equipe, pois articula e interage com os diferentes trabalhadores e é identificado pela liderança e coordenação do processo de trabalho em saúde. Contudo, no desempenho desse papel, esse profissional defronta-se com a necessidade de gerenciar conflitos, o que tem sido referido como uma fonte de sofrimento moral ${ }^{(9-10)}$.

Para minimizar esse sofrimento, alguns autores mencionam aspectos essenciais para propiciar a resolução dos conflitos, dentre os quais se destacam a formação básica adequada e a utilização de instrumentos gerenciais, como planejamento, liderança, autonomia e administração adequada do tempo ${ }^{(11-12,26)}$. Estudos complementam, destacando como principais competências do enfermeiro gerencial: comunicação, flexibilidade, tomada de decisão e visão estratégica, gerenciamento de pessoas, materiais e custo hospitalar, relacionamento interpessoal e trabalho em equipe ${ }^{(15,27)}$.

No que diz respeito à comunicação e ao trabalho em equipe, observou-se que exigem do enfermeiro a aquisição de outras competências ou capacidades, para que possa agir com efetividade na prática hospitalar cotidiana. A esse respeito, sabe-se que a mediação de conflitos é uma das capacidades essenciais ao bom andamento do trabalho de toda equipe ${ }^{(28)}$.

Os conflitos são comuns entre os seres humanos. Constituem-se em discordâncias resultantes de contestações de valores ou anseios que provocam o rompimento da ordem. Podem ser funcionais, de caráter construtivo, ou disfuncionais, de cunho negativo, afastando aqueles que o percebem ${ }^{(29)}$. Independente da habilidade profissional, os conflitos podem levar a angústias frequentes, desavenças e insatisfação no trabalho, tornando o clima hostil ${ }^{(18)}$. A mediação pode contribuir para minimizar insatisfações e desmotivação, mantendo a harmonia e o equilíbrio da equipe, o que reflete positivamente em todo o cuidado prestado ${ }^{(28)}$.

Para se administrar os conflitos nas unidades assistenciais, é essencial conhecer a sua origem, visto que não há protocolos determinados para esse fim. Muitas vezes, surgem de problemas relacionados ao tipo de diálogo, de estrutura organizacional e comportamento individual. Para resolvê-los, é necessário desenvolver planos de ação que compreendam todas as partes envolvidas e reconheçam as diferenças entre as pessoas, pois todo ser humano é um ser único ${ }^{(30-32)}$.

Ressalta-se que o enfermeiro é o líder da gestão de pessoas, pois interage direta e sucessivamente com a equipe de trabalho. Sendo assim, não só o gestor de enfermagem, mas cada enfermeiro, como líder, também é responsável pela administração do recurso humano na organização. Todavia, saberes sobre liderança e administração são essenciais à gestão da instituição, independentemente da área de ocupação. Diante disso, o profissional responsável pela gerência do processo deve possuir competências, habilidades e atitudes assertivas para gerenciar conflitos ${ }^{(17)}$.

Desse modo, o enfermeiro gestor deve ser dotado da aptidão de liderança para gerenciar conflitos. Para tanto e para atender às exigências das instituições, que requerem postura ética e atitude do enfermeiro para atingir objetivos e excelência no trabalho em equipe, a comunicação interpessoal é crucial $^{(33)}$.

Ressalta-se a importância dos centros formadores, no que tange à formação desses profissionais. É indispensável a implementação de estratégias para desenvolver competências clínicas e gerenciais, fortalecendo, nos discentes, as capacidades profissionais indispensáveis 
para a apropriada atuação frente à integralidade do cuidado ${ }^{(13-14,18,20,30)}$.

\section{Estratégias negociadoras na resolução de conflitos}

$\mathrm{Na}$ contemporaneidade, os ambientes estão cotidianamente mais competitivos e com maior pressão, o que favorece o surgimento de conflitos. Diante dessa realidade, as mudanças do modelo de atenção requerem o empenho no desenvolvimento de boas práticas de diálogo, relacionamento e gestão interpessoais. Essa mudança, a cada dia, distancia-se mais do modelo médico hegemônico, acolhendo novos estilos de fazer saúde que concorrem para o fortalecimento e a confiabilidade nas atuações das práticas assistenciais e gerenciais do enfermeiro ${ }^{(18)}$.

Rotineiramente, ressalta-se o tempo gasto pelos enfermeiros líderes na resolução dos conflitos, que nem sempre atendem às expectativas das partes, e elas deem-se por satisfeitas. Seja qual for o tipo de conflito, acredita-se que o diferencial de uma boa resolutividade esteja ligado diretamente a uma tática consciente, em que seja estabelecida a mediação por meio da comunicação e da escuta.

Para uma boa resolutividade dos conflitos, é necessário conhecê-los. Dentre os diversos tipos existentes, sobressaem-se: conflito latente, que é o conflito não declarado, não existindo uma clara consciência dos envolvidos, eventualmente não sendo necessário intervir; conflito percebido, em que há existência e percepção do conflito, porém não há manifestação aberta desse; conflito sentido, aquele que já atinge ambas as partes, e em que há emoção e forma consciente; e há conflito manifesto, que atinge todas as partes, é nítido para terceiros e pode prejudicar a dinâmica da equipe ${ }^{(1)}$.

Desse modo, a mediação destaca-se como um processo de intervenção sobre os diversos tipos de conflitos e deve ser utilizada de modo a buscar uma solução aceitável para os envolvidos, contribuindo para a redução dos impactos negativos e, particularmente no ambiente hospitalar, para a manutenção de relações baseadas em respeito e confiança ${ }^{(34)}$.

A escolha da estratégia mais adequada para a resolução de conflitos depende de várias situações, tais como: a praticidade em se resolver o problema, a individualidade dos envolvidos e a relevância da questão ${ }^{(35)}$. Dentre as estratégias resolutivas de conflito, destacam-se: comunicação efetiva e feedback com a equipe multidisciplinar; capacidade de observação, o que requer do enfermeiro uma visão do todo, mas com o foco no cumprimento de normas e rotinas; discernimento na compreensão das diversas situações, sendo a ética e a discrição imprescindíveis nesse processo. Acrescentam-se o comprometimento, uma vez que, ao envolver toda a equipe, o trabalho torna-se mais prazeroso, e a liderança estratégia indispensável, pois a postura do líder conduz a equipe de maneira positiva e satisfatória e a envolve em todos os processos com responsabilidade ${ }^{(36)}$.

Outros estudos reiteram como estratégias eficazes a serem utilizadas pelos enfermeiros para a resolução de conflitos: o atendimento de qualidade ao usuário, a comunicação eficaz, o saber trabalhar em equipe, ser ético, ter boa postura, saber ouvir, reconhecer seu papel diante da equipe ${ }^{(23-25)}$.

Salienta-se o dimensionamento de pessoal como uma estratégia de competência do enfermeiro para a resolução de conflitos, visto que, quando realizado de forma adequada, pode minimizar a sobrecarga de trabalho, valorizar o trabalho do outro e, consequentemente, reduzir os conflitos ${ }^{(19,21)}$.

Reafirma-se que a liderança do enfermeiro é fundamental na gerência de conflitos, pois o líder deve assumir a comunicação integrada eficaz, ser responsável pela coordenação e negociação, como forma de prevenção interdisciplinar de conflitos; deve ainda ter visão ampla e caráter flexível no enfrentamento de hostilidades, com vistas a manter o ambiente de trabalho propício, saudável e harmonioso, para proteger a qualidade do serviço de saúde ${ }^{(11,24)}$. 
Considera-se que o sucesso da mediação e do conhecimento técnico, já mencionados como práticas recomendadas para conduzir e resolver conflitos, identifica-se também com a importância do diálogo, quando há o envolvimento de usuários e seus familiares ${ }^{(37)}$.

Com o conhecimento dos tipos de conflitos é essencial deter a habilidade de mediá-los. Essa habilidade é essencial quando aplicada com efetividade, a fim de ser resolutiva e justa. Logo, o mediador deve ter consigo alguns princípios: possuir credibilidade, ser imparcial, estar ciente da situação, ser maleável em suas decisões e o mais transparente possível, mantendo-se sempre ético ${ }^{(28)}$.

Dentre as limitações deste estudo, destaca-se o período reduzido de busca de artigos e restrição de seleção a apenas três idiomas. Assim, recomenda-se a ampliação para períodos maiores e com outros idiomas, de forma a melhor contribuir para aprofundar o conhecimento sobre a temática. Destaca-se que este estudo pode contribuir com reflexões sobre o ambiente harmonioso da assistência de enfermagem, ao permear situações complexas, em que saber negociar conflitos trará resultados positivos tanto para o cuidado prestado ao usuário dos serviços quanto para a instituição.

\section{Conclusão}

Este estudo permitiu a identificação de publicações científicas atuais que abordaram a temática gerenciamento de conflitos, enfatizando a capacidade de negociação como uma das competências do enfermeiro no contexto do hospital, com vistas a organizar o processo de trabalho e trazer benefícios para a organização.

Compreende-se que os artigos aludiram diversos tipos de conflitos e que eles são inerentes à interação de recursos humanos com o ambiente de trabalho, cabendo ao enfermeiro, responsável pela equipe, gerenciá-los, a fim de promover um ambiente adequado de trabalho, motivador ao trabalhador para boas práticas de enfermagem.
Os estudos selecionados apresentaram as dificuldades do enfermeiro, enquanto gestor do cuidado, em relação à competência para gerenciar conflitos, pois nem sempre ambos os lados envolvidos conseguem ficar satisfeitos com as resoluções propostas. Para viabilizar essa tarefa, são necessárias estratégias de gerenciamento, destacadas neste estudo: saber liderar, negociar, planejar, ser justo frente às situações, avaliar, ter conhecimento científico e técnico para, assim, conseguir conduzir a equipe. É preciso ainda conhecer a equipe, suas particularidades e a personalidade de cada trabalhador, para evitar novas ocorrências de conflitos, tendo em vista o objetivo maior do cuidado de qualidade ao usuário.

Reitera-se que o comportamento do enfermeiro, diante de uma situação conflituosa, depende de seu conhecimento, habilidades e atitudes enquanto gerente, das suas aptidões pessoais no processo de negociação e utilização da estratégia mais propícia para resolver ou diminuir os conflitos que ocorrem no trabalho.

As instituições formadoras em Enfermagem têm a responsabilidade de implementar estratégias ou metodologias inovadoras com foco no desenvolvimento de competências gerenciais ainda no âmbito da graduação, como o gerenciamento de conflitos, necessárias às demandas do mercado de trabalho em saúde.

\section{Colaborações:}

1 - concepção, projeto, análise e interpretação dos dados: Denise Maria Osugui, Silvia Helena Henriques, Eliza Maria Rezende Dázio, Zélia Marilda Rodrigues Resck, Laura Andrian Leal e Roberta Seron Sanches;

2 - 2redação do artigo e revisão crítica relevante do conteúdo intelectual: Denise Maria Osugui, Silvia Helena Henriques, Eliza Maria Rezende Dázio, Zélia Marilda Rodrigues Resck, Laura Andrian Leal e Roberta Seron Sanches;

3 - aprovação final da versão a ser publicada: Denise Maria Osugui, Silvia Helena Henriques, Eliza Maria Rezende Dázio, Zélia Marilda Rodrigues Resck, Laura Andrian Leal e Roberta Seron Sanches. 


\section{Referências}

1. Teixeira NL, Silva MM, Draganov PB. Desafios do enfermeiro no gerenciamento de conflitos dentro da equipe de enfermagem. Rev Adm Saúde. 2018;18(73). DOI: http://dx.doi.org/10.23973/ras. 73.138

2. Barbiani R, Nora CRD, Schaefer R. Nursing practices in the primary health care context: a scoping review. Rev Latino-Am Enfermagem. 2016;24:e2721. DOI: http://dx.doi.org/10.1590/1518-8345.0880.2721

3. Karami A, Farokhzadia J, Foroughameri G. Nurses' professional competency and organizational commitment: is it important for human resource management? PLoS One. 2017;12(11):e0187863. DOI: http://dx.doi.org/10.1371/journal.pone.0187863

4. Maia NMFS, Fonseca BAV, Coelho LS, Carvalho Junior JAM, Maia SF, Andrade EWOF. Percepção da equipe de enfermagem sobre a função do gerente de enfermagem hospitalar. $\mathrm{R}$ pesqui (Univ Fed Estado Rio J, Online). 2020 jan-dez;12:1-5. DOI: 10.9789/2175-5361.rpcfo. v12.6555

5. Copelli FHS, Erdmann AL, Santos JLG. Entrepreneurship in Nursing: an integrative literature review. Rev Bras Enferm. 2019;72 (Suppl 1):289-98. DOI: http://dx.doi.org/10.1590/ 0034-7167-2017-0523

6. Felix AMS, Toffolo SR. Participation of nurses in antimicrobial stewardship programs: an integrative review. Cogitare enferm. 2019;24:e59324. DOI: http://dx.doi.org/10.5380/ce.v24i0.59324

7. Tostes MFP, Galvão CM. Implementation process of the Surgical Safety Checklist: integrative review. Rev Latino-Am Enfermagem. 2019;27:e3104. DOI: http://dx.doi.org/10.1590/1518-8345.2921.3104

8. Melnyk BM, Fineout-Overholt E. Evidence-based practice in nursing and helthcare: A guide to best practice. 4nd ed. China: Wolters Kluwer; 2019.

9. Ramos FRS, Vargas MAO, Schneider DG, Barlem ELD, Scapin SQ, Schneider AMM. Ethical conflict as a trigger for moral suffering: survey of Brazilian nurses Rev enferm UERJ. 2017;25:e22646. DOI: http://dx.doi.org/10.12957/reuerj.2017.22646

10. Wood-Molina T, Rivas-Ribeiro E. Conflictividad ética en enfermeras/os de unidades de cuidados críticos en un hospital del sur de Chile. Enfermería Universitaria. 2017;14(4):224-34. DOI: https://doi. org/10.1016/j.reu.2017.09.002
11. Eduardo EA, Peres AM, Kalinowski CE, Cunha ICKO, Bernardino E. The negotiator that we have and the negotiator that we want in nursing. Texto contexto-enferm. 2016;25(3):e1030015. DOI: http://dx.doi.org/10.1590/0104-07072016001030015

12. Pinhatti EDG, Vannuchi MTO, Sardinha DSS, Haddad MCL. Job rotation of nursing professionals among the sectors of a hospital: a management tool in conflict resolution. Texto Contexto Enferm. 2017;26(2):e1180015. DOI: http://dx.doi. org/10.1590/0104-07072017001180015

13. Leal LA, Soares MI, Silva BR, Brito LJS, Bernardes A, Henriques SH. Professional competencies for hospital nurses: a documentary analysis. Rev Enfermagem Centro-Oeste Mineiro. 2019;9:e3249. DOI: http://dx.doi.org/10.19175/ recom.v9i0.3249

14. Camelo SHH, Soares MI, Chaves LDP, Rocha FLR, Silva VLS. Nurse managers at a teaching hospital: training, responsibilities and challenges. Rev enferm UERJ. 2016;24(3):e11637. DOI: http:// dx.doi.org/10.12957/reuerj.2016.11637

15. Leal LA, Soares MI, Silva BR, Bernardes A, Camelo SHH. Clinical and management skills for hospital nurses: perspective of nursing university students. Rev Bras Enferm. 2018;71(Suppl 4):1514-21. DOI: http://dx.doi.org/ 10.1590/0034-7167-2017-0452

16. Tironi NM, Bernardino E, Haddad MCL, Nimtz MA, Torres DG, Peres AM. Assignments and competencies of nursing managers: a descriptive exploratory research. Online braz j nurs [Internet] 2017 [cited 2020 May 20];16(2):130-9. Available from: http://www.objnursing.uff.br/index.php/ nursing/article/view/5601

17. Grubaugh ML, Flyn L. Relationships Among Nurse Manager Leadership Skills, Conflict Management, and Unit Teamwork. J Nurs Adm. 2018;48 (7-8):383-8. DOI: http://dx.doi.org/10.1097/NNA. 0000000000000633

18. Beserra EP, Gubert FM, Martins MC, Vasconcelos VM, Figueiredo GA, Silva LA, et al. Conflict management in nurse training. J Nurs UFPE online. 2018;12(10):2891-6. DOI: https://doi.org/10.5205/1981-8963-v12i10a236080p28912896-2018

19. Souza MS, Barlem JGT, Hirsch CD, Rocha LP, Neutzling BRS, Ramos AM. Dimensionamento e escalas de pessoal de enfermagem: competências dos enfermeiros. Enferm Foco. 
2018;9(2):50-5. DOI: https://doi.org/10.21675/2357707X.2018.v9.n2.1072

20. Amestoy SC, Peixoto RS, Garcia RP, Santos BP, Silva CN, Braga DD. Percepção de enfermeiroslíderes sobre o gerenciamento de conflitos no ambiente hospitalar. Rev enferm UFSM. 2016;6(2):259-69. DOI: http://dx.doi.org/10.5902/ 2179769218167

21. Cordero-Maldonado E, García-Domínguez JA, Romero-Quechol GM, Flores-Padilla L, Trejo-Franco J. Dimensiones de la relación interpersonal del profesional de enfermería en una unidad de segundo nível. Rev Enferm Inst Mex Seguro Soc [Internet]. 2019 [cited 2019 Dec 12];27(2):89-96. Available from: https://pesquisa. bvsalud.org/portal/resource/pt/biblio-1015232

22. Santos JLG, Lima MADS, Pestana AL, Colomé ICS, Erdmann AL. Estratégias utilizadas pelos enfermeiros para promover o trabalho em equipe em um serviço de emergência. Rev Gaúcha Enferm. 2016;37(1):e50178. DOI: https:// doi.org/10.1590/1983-1447.2016.01.50178

23. Thofehrn MB, Montesinos MJL, Jacondino MB, Fernandes HN, Gallo CMC, Figueira AB. Work process of nurse in health production in a university hospital in Múrcia/Espanha. Cienc Cuid Saude. 2015;14(1):924-32. DOI: https://doi. org/10.4025/cienccuidsaude.v14i1.22094

24. Ika C, Novieastari E, Nuraini T. The role of a head nurses in preventing interdisciplinary conflicts. Enferm Clin. 2019;(Suppl 2):123-7. DOI: https://doi.org/10.1016/j.enfcli.2019.04.019

25. Kim WS, Nicotera AM, McNulty J. Nurses' perceptions of conflict as constructive or destructive. J Adv Nurs. 2015;71(9):2073-83. DOI: https://doi.org/10.1111/jan.12672

26. Jiménez-Gómez MA, Cárdenas-Becerril L, Velásquez-Oyola MB, Carrillo-Pineda M, Barón-Díaz LY. Reflective and critical thinking in nursing curriculum. Rev Latino-Am Enfermagem. 2019;27:e3173. DOI: 10.1590/1518-8345.2861.3173

27. Lessa JD, Silva AR. Competências gerenciais: um desafio para a enfermagem. Rev Científica Multidisciplinar Núcleo Conhecimento [Internet]. 2017 [cited 2019 Dec 12];1(2):60-71. Available from: https://www.nucleodoconhecimento.com. $\mathrm{br} /$ saude/competencias-gerenciais

28. Lopes ER, Souza JEO, Almeida MLS, Silva WA. Conflitos na equipe de enfermagem: um desafio na gestão do enfermeiro. Única cad acad [Internet]. 2015 [cited 2019 Dec 12];1(1):1-10. Available from: http://co.unicaen.com.br:89/periodicos/index. $\mathrm{php/UNICA/article/viewFile/10/10}$

29. Marques FRB, Ferreira MCV, Duarte AM, Balieiro MMFG, Mandetta MA. Nature and source of conflicts of relationships in the context of pediatric oncology: an integrative literature review. Ciênc Cuidado Saúde. 2015;14(2):1184-93. DOI: https:// doi.org/10.4025/cienccuidsaude.v14i2.25247

30. Lanzoni GMM, Meirelles BHS, Erdmann AL, Thofehrn MB, Dall'agnol CL. Actions/interactions motivating nursing leadership in the context of primary health care. Texto contexto enferm. 2015;24(4):1121-9. DOI: http://dx.doi.org/ 10.1590/0104-0707201500003740013

31. Oliveira ES, Cruz KG, Almeida HOC, Melo IA, Góis RMO. A gestão de conflitos: desafio na prática gerencial do enfermeiro. Ciênc Biol Saúde Unit [Internet]. 2018 [cited 2019 Dec 12];4(3):123-38. Available from: https://periodicos.set.edu.br/ index.php/cadernobiologicas/article/view/5162

32. Bristot RB, Ceretta LB, Soratto MT. Conflitos éticos da equipe de enfermagem no processo de trabalho na atenção básica. Enfermagem Brasil [Internet]. 2017 [cited 2019 Dec 12];16(1):11-9. Available from: https://portalatlanticaeditora.com.br/index. php/enfermagembrasil/article/view/899/1853

33. Silva JCB, Silva AAOB, Oliveira DAL, Silva CC, Barbosa LMS, Lemos MEP, et al. Profile of the nurse in the management of hospital services. J Nurs UFPE online. 2018;12(10):2883-90. DOI: https://doi. org/10.5205/1981-8963-v12i10a236307p2883-2890-2018

34. Claro RFS, Cunha PFSS. Estratégias de gestão construtiva de conflitos: uma perspectiva dos profissionais de saúde. Psicol Saúde Doenças. 2017;18(1):55-68. DOI: http://dx.doi. org/10.15309/17psd180105

35. Ahanchian MR, Emami-Zeydi A, Armat MR. Conflict Management Styles Among Iranian Critical Care Nursing Staff: a cross-sectional study. Dimens Crit Care Nurs. 2015;34(3):140-5. DOI: http://dx.doi. org/10.1097/DCC.0000000000000106

36. Souza ACD, Silva FS, Espindola JS, Moreira NL, Draganov PB. Atuação do enfermeiro nas estratégias de resolução de conflitos. Rev Adm Saúde. 2018;18(73). DOI: http://dx.doi. org/10.23973/ras.73.142 
37. Nascimento D. Mediação de Conflitos na Área

Recebido: 26 de março de 2020 da Saúde: experiência portuguesa e brasileira. Cad Ibero-Amer Dir Sanit. 2016;5(3):201-11. DOI: http://dx.doi.org/10.17566/ciads.v5i3.333

Aprovado: 8 de maio de 2020

Publicado: 14 de julho de 2020

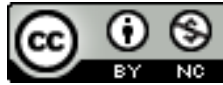

A Revista Baiana de Enfermagem utiliza a Licença Creative Commons - Atribuição-NãoComercial 4.0 Internacional. https://creativecommons.org/licenses/by-nc/4.0/ Este artigo é de acesso aberto distribuído sob os termos da Licença Creative Commons (CC BY-NC). Esta licença permite que outros remixem, adaptem e criem a partir do seu trabalho para fins não comerciais. Embora os novos trabalhos tenham de lhe atribuir o devido crédito e não possam ser usados para fins comerciais, os usuários não têm de licenciar esses trabalhos derivados sob os mesmos termos. 